\title{
A location-based MAC protocol for safety-of-life vehicle-to-vehicle communication
}

\author{
C. R. Garcia, T. Strang \& A. Lehner \\ German Aerospace Center (DLR), \\ Institute for Communications and Navigation, 82234 Wessling, Germany
}

\begin{abstract}
Safety of life applications have high importance in today's traffic as current statistics show. In particular active safety applications are at the center of research activities, focussing on vehicle to vehicle communications as it is easier and cheaper to deploy as well as more effective in most situations. This approach has also to be followed for railway traffic. However, different vehicle types have different characteristics and therefore different requirements to their communication systems and the communication networks in particular. We identified here the need for an adapted Medium Access Control (MAC) protocol, called the Cell-based Orientation-aware MANET Broadcast (COMB) protocol, that fulfills the requirements of the train specific scenario and overcomes the drawbacks of all existing approaches. COMB is based on localization aware cross layer dimensioned CDMA cells, and uses the SOTDMA protocol as am intra cell scheme, while the inter cell scheme relies on direction and speed awareness. Therefore, it can cope with the trains' speed and their need for a robust communication.
\end{abstract}

\section{Introduction}

Current statistics of the International Union of Railways (UIC) show that there are three significant train accidents in Europe every day [1], despite millions of Euros which have been invested in trackside and in-train safety equipment. In order to increase safety in railway traffic, some countries are partially installing control systems, mainly centralized management ones, specially the Automatic Train Control (ATC), where the trains are monitored by devices located along the rail. These devices send the collected information to an operation center that can pass specific instructions to the train. A European ATC standard, European Train Control Sys- 
tem (ETCS), is intended to replace the various European ATC systems, in order to protect international train traffic. However according to the estimations of the German railway company "Deutsche Bahn" (DB), it could take up to 20 years and cost up to 8 billion Euros to introduce ETCS right across Europe [2]. Additionally, only the operation center has an overall overview of the traffic situation, and a train driver could only be warned of a hypothetical collision if the operation center decides it.

While maritime, air, and road transport have a vehicle integrated collision avoidance system available or in the development phase, we find no satisfactory solution of this type of technology in railway transportation.

Therefore the German Aerospace Center (DLR) is currently developing a Railway Collision Avoidance System (RCAS) [3] that will allow the drivers to have an up-to-date accurate knowledge of the traffic situation in the vicinity, and act in consequence. The system is intended to not rely on components in the infrastructure, this way substantially reducing its rollout and maintenance costs, as well as inherently providing a migration strategy. The basic idea of RCAS is to calculate the own position and movement vector and broadcast this information as well as additional data like vehicle dimensions to all other trains in the area. Thus, the driver's cabin could be equipped with a display showing the position of the other vehicles in the region. Computer analysis of the received information, the own position and movement vector and an electronic track map detects possible collisions, displaying an alert signal, and advising the driver of the most convenient strategy to follow in order to avoid the danger. The system can take into account different danger sources, like advancing trains or road vehicles or obstacles, and classify them according to a specific scale.

This system presents many technical challenges on the design of the communication approach. In this paper we will focus on the Medium Access Control (MAC) layer. This layer coordinates packets transmissions. Since RCAS should be an infrastructure-less broadcast system where the trains are moving at high speeds, the network configuration is high dynamic "mobile ad-hoc network" (MANETs).

The paper is organized as follows: First, the requirements of the RCAS system are discussed. Section 3 examines the MAC layers used in other transportation system. Then, their appropriateness for RCAS are analyzed and their problems are pointed out. Section 4 presents the Cell-based Orientation-aware MANET Broadcast (COMB) protocol, a protocol that can be used in vehicle to vehicle communication and that overcomes the problems mentioned in Section 3. Finally, Section 5 provides a summary and an outlook.

\section{System requirements}

In order to get a better picture of how the MAC layer should look, the requirements of the system will be analyzed. In particular we will consider:

- Range: In order to allow for a sufficiently long braking distance (measured from the moment when the train detects, that there is an hazardous situation, until it has stopped completely) the range should be in the order of $5 \mathrm{~km}$. 
- Speed: speeds up to $300 \mathrm{~km} / \mathrm{h}$ should be supported.

- Message Rate: The message rate should be about one second.

- Number of users: The protocol should be able to cope with 100 trains in its range.

In RCAS, the MAC layer design depends strongly on the speed of the trains, parameters like message rate and maximum density are inferred from this value. This way, high speeds require a larger message rate, since the position information should be updated more frequently.

Since multihop and addressed communication are not foreseen in a first RCAS approach, there is no network layer. Therefore, there is no upper layer after the MAC layer to manage packet collisions and thus, the MAC layer should avoid packet collision or ensure a suitable low level of collision rate for a surveillance application.

Furthermore, the RCAS MAC layer should be capable of operating without the support of fixed infrastructure. Therefore, a distributed protocol should be used. This way, the structure of the network is ad-hoc.

On the other hand, the protocol should be suited for broadcast communications, for it cannot assume a prior knowledge of the current network configuration. Retransmissions of the packets will not be considered, since the retransmitted information would be outdated. Waiting for the next updated message outweighs the retransmission option. Consequently the protocol does not have to deal with Acknowledgment (ACK) messages.

\section{MAC layer in VANETs}

In the previous section it has been exposed which are the desired characteristics of a MAC layer for RCAS. In this section we will study the MAC layers used in collision avoidance systems that conform vehicle broadcast ad-hoc networks. In particular, Automatic Identification System (AIS) [4] for maritime transportation (Figure 1), Traffic Alert and Collision Avoidance System/Automatic Dependent Surveillance-Broadcast (TCAS/ADS-B) [5] for air transportation (Figure 2) and Car-2-Car [6] for road transportation (Figure 3).

\subsection{Maritime navigation}

Maritime AIS should be capable of operating autonomously on the high seas. Consequently a distributed protocol is utilized. The technology used for this purpose relies on a protocol called Self Organized Time Division Multiple Access (SOTDMA) [7], where the data stream is placed in defined time slots. Depending on the observed data traffic, each AIS module builds its own timetable for data transfer and reserves free time slots for its messages. In order to ensure synchronized time slots, a very precise clock is needed. Since the navigation equation provides position and time information, the GPS receiver assures the required time accuracy [8]. Coming up to the length and message rate, AIS slots are 256 bits long and it supports 4500 reports per minute. Message rate depends on the own speed, the values go from $2 \mathrm{~s}$ for speeds over 23 knots, to 3 min for speeds under 3 knots, leading 


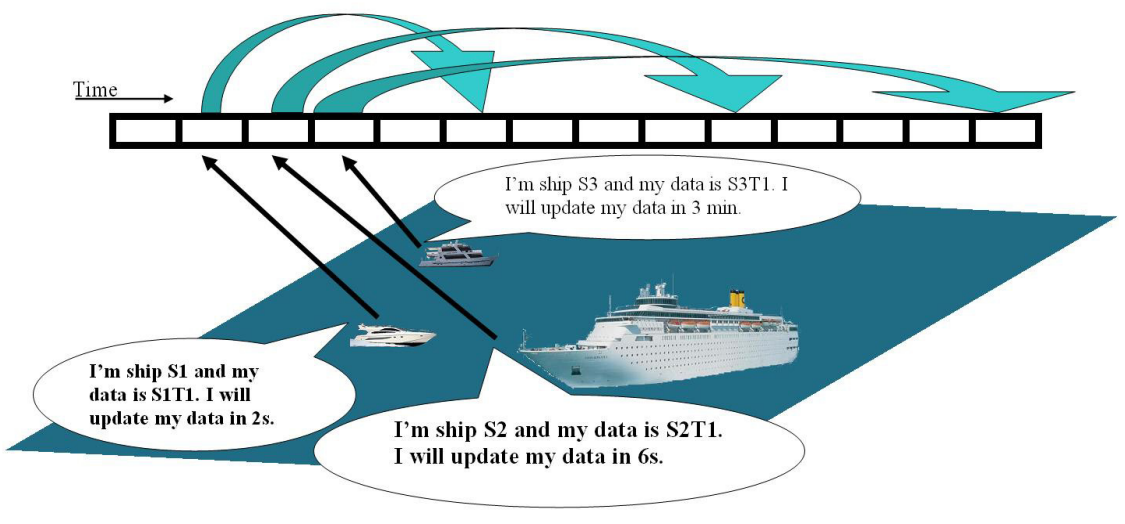

Figure 1: AIS surveillance strategy.

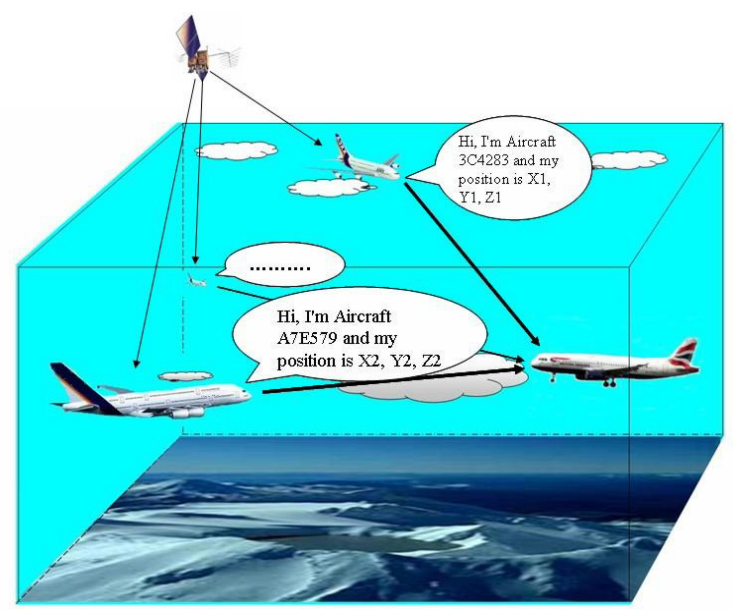

Figure 2: ADS-B surveillance strategy.

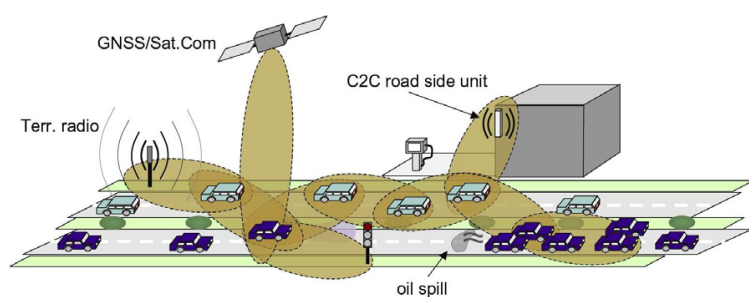

Figure 3: Car-2-car surveillance strategy. 
to a density of around 400 ships in range. In order to maintain the density below SOTDMA is able to reduce the range radius in necessary.

\subsection{Aviation}

Despite its low throughput, TCAS MAC protocol is based on Aloha. However, colliding transmissions can be greatly diminished by means of different techniques, i.e, interference limiting, passive detection, altitude comparison, interrogation frequency, and directional antenna. Every TCAS unit monitors the number of TCAS units within the detection range. This information is used to limit its own interrogation rate and power as necessary. In order to minimize the number of interrogations, the identity and altitude of targets is intended to be determined by passively monitoring transmissions received, afterwards, error detection and correction is applied to the received messages to reduce the number of erroneous addresses to be processed. Nonthreat intruded are dismissed through altitude comparison. Additionally, TCAS units transmit an interrogation sequence nominally once per second for only those intruders that could become immediate threats. Intruders that are not likely to become immediate threats should be interrogated less frequently. Differentiation is made by means of range and estimated range rate. Finally, a directional antenna sequentially generates beams that point in the forward, aft, left and right direction. Together these beams provide surveillance coverage for targets at all azimuth angles, this way synchronous garble is reduced, i.e, the side lobe signals are ignored. On the other hand, a directional interrogation reduces the size of the interrogation region. Thus, minimizing the number of transmission collisions. Message length are 56 or 112 bits while the nominal density is 30 aircraft in range.

Similarly to TCAS, ADS-B is based on Aloha. In this case, interference limitation is as well applied. Since the ADS-B extended squitter includes additionally position and speed information, its length is 112 bits instead the 56 bits of the TCAS squitter, where only identity information is transmitted.

\subsection{Road transport}

For C2C, a derivate of IEEE 802.11 standard and CSMA/CA (Carrier Multiple Access with Collision Avoidance) protocol in the MAC layer are proposed. One of the main limitations is the large amount of data stored by the multiple sensors it uses. Therefore, the message length is so large that the network might collapse. In order to reduce the length of the message, the inferred information of the combined sensors' output might be sent, instead of transmitting the direct output of the sensor.

\subsection{Applicability to RCAS}

Very different approaches are carried out for each one of the analyzed systems. While AIS and C2C have well defined and standardized MAC protocols, ADSB and TCAS utilize an "statistical" approach. However, they all share a common feature, the MAC protocols are distributed in order to be managed autonomously. Consequently, the RCAS MAC layer will be distributed. 
The drawback of Aloha is its low throughput, it is extremely sensitive in high density situations like in shunting yards or train stations. However, this protocol simplifies considerably the MAC layer.

Nonetheless, SOTDMA and CSMA/CA are not suitable protocols for RCAS. CSMA/CA uses the mechanism RTS/CTS (Request to send/Clear to send) to avoid the hidden terminal problem [9]. Although it is specially designed for wireless networks, it does not work properly in broadcast communication, since the CTSs packets would collide, due to the non defined number of recipient and the disparity of the reception conditions between them. On the other hand SOTDMA does not solve the hidden terminal problem and does not assure absence of transmission collision as a contention phase exists, when a newcomer enters the network and tries to occupy a free time slot. Therefore, in the case two newcomers are simultaneously entering and by chance both occupy a free time block a transmission collision occurs. This type of collision could hardly occur if the ad-hoc networks are stable, i.e., the host's situation in the network changes slowly. On the other hand, if too many hosts are leaving and entering continuously the network, the number of packet collisions might increase significantly. Since ships have a relative low speed and course change, SOTDMA works properly in AIS. Nonetheless, in RCAS, due to the potential high speed of trains and the relative low communication range, the network is more unstable and therefore, the protocol might be not suitable.

Aircraft are able to move in any direction, having more reaction possibilities, which leads to a lower message rate compared to trains. However, the speed of aircraft is higher. Therefore, the reaction time is reduced, and consequently, the message rate is increased. Summing up, the message rate for trains is expected to be in the range of the one for aircraft. As shown in Tables 1 and 2, RCAS has very restrictive conditions due to the relationship between speed and range, the network configuration changes very rapidly. Therefore, an optimum protocol should be designed that overcomes the problems of the protocols described in this section.

Table 1: Range in the collision avoidance systems.

\begin{tabular}{|l||l|}
\hline AIS & $28-55 \mathrm{~km}$ \\
\hline ADS-B & $<370 \mathrm{~km}$ \\
\hline Car2Car & $1 \mathrm{~km}$ \\
\hline RCAS & $5 \mathrm{~km}$ \\
\hline
\end{tabular}

Starting from the SOTDMA protocol, we have developed a protocol called Cellbased Orientation-aware MANET Broadcast (COMB) [10]. COMB overcomes the two main drawbacks from SOTDMA:

1. The hidden terminal problem: It assures that vehicles that can not see each other use different channels, concretely CDMA codes. While vehicles in range use the same code but are differentiated through SOTDMA. In order 
Table 2: Speed in the collision avoidance systems.

\begin{tabular}{|l||l|}
\hline AIS & $70 \mathrm{~km} / \mathrm{h}$ \\
\hline ADS-B & $700 \mathrm{~km} / \mathrm{h}$ \\
\hline Car2Car & $150 \mathrm{~km} / \mathrm{h}$ \\
\hline RCAS & $300 \mathrm{~km} / \mathrm{h}$ \\
\hline
\end{tabular}

to accomplish this effect, the communication area is geographically divided in hexagonal cells with one of 12 different codes associated to it. All the vehicles located in a cell transmit their messages with the cell code and use the SOTDMA frame.

2. Collision during contention: The vehicles crossing to a new cell, have to access the SOTDMA structure valid in the area, a priority protocol is applied in order to avoid packet collisions.

\section{Cell-based orientation-aware MANET broadcast MAC layer}

In this section we will finally present the specification of the COMB protocol:

1. Divide the map in hexagonal cells where its dimension is given by equation (1).

$$
D=R-2 * F
$$

where $R$ is the minimum range and $F$ is the distance of a slot frame. $F$ can be calculated as:

$$
F=v * t
$$

where $v$ is the maximum speed of the node and $t$ is the minimum time between two consecutive messages of a node. For example, if the nodes have a range of $7 \mathrm{~km}$, the messages are sent each second, and it is moving at $300 \mathrm{~km} / \mathrm{h}$, then in order to receive two slot frames before crossing to the new cell $2 * F=(300 * 1000) * 2 / 60 * 60=0,167 \mathrm{~m}$

2. Give each cell a code of a group of twelve orthogonal CDMA codes in such a way that there are three cells between two cells with same code.

3. Inside a cell the messages are codified with the corresponding cell code.

4. Inside a cell the messages are sent according the SOTDMA protocol. The time is divided in "slot frames" where the frame duration is the minimum time between two consecutive messages coming from a same node.

5. The nodes observe the "slot frames" codified with its cell code. The nodes send their messages in their already reserved slots and reserve a free slot in a next "slot frame".

6. The nodes observe the "slot frames" codified with its cell code. The nodes send their messages in their already reserved slots and reserve a free slot in a next "slot frame". 
7. The cell priority is given by the position of the current cell with respect to the target cell. The highest priority corresponds to the cell in the north.

\section{Conclusion and outlook}

In this paper we explained, why there is a need for a vehicle-to-vehicle communication based collision avoidance system in railway traffic. At the roots of such a system is a working, reliable communication network. We focussed here on the tasks and gains by MAC layers in general before we presented our COMB protocol for broadcast MANETs.

COMB divides the network in cells characterized by one CDMA code while within each cell an SOTDMA protocol is used. Our protocol cooperates with the physical layer in order to control the range. The protocol is orientation aware and using data from a Global Navigation Satellite System (GNSS) such as GPS. Positioning is necessary in order to recognize in which cell the vehicle is and which CDMA code should utilize. Speed and direction information are used when moving to another cell. Like that we can avoid the hidden terminal problem and collisions due to contention in control as well as data Channels with only 12 orthogonal codes and a minimum of overhead.

Our future work regarding the communication network has two steps. The above presented approach has to be simulated and evaluated thoroughly, as well as compared to existing ones and different options in this one (for instance frequency division instead of code division for cells). This step completed we will investigate in detail the physical layer. Having already examined the channel model [11], we will have to apply these insights into our system and verify them by simulations and tests.

\section{References}

[1] Safety Database Project Team (UIC-SDB), State of the art, the UIC safety data base (UIC-SDB), 2006.

[2] Schmid, G., Risk management in rail transportation, Casualty Risk Consulting Information for insurers, Mnchener Rckversicherungs-Gesellschaft, 2006.

[3] Strang, T., Meyer zu Hrste, M. \& Gu, X., A railway collision avoidance system exploiting ad-hoc inter-vehicle communications and galileo. Proceedings, 13th World Congress and Exhibition on Intelligent Transportation Systems and Services (ITS 2006), 2006.

[4] AIS official website. http://www.navcen.uscg.gov/enav/ais/default.htm.

[5] International standards and recommended practices. aeronautical telecommunications. annex 10, to the convention on international civil aviation. volume iv surveillance radar and collision avoidance systems, 2005.

[6] Car-2-car communication consortium home page.

[7] van der Heijden, W., AIS is coming! ION GPS 2001, 2001. 
[8] Hakam Lans, Saltsjbaden, Position indicating system. United States Patent, Patent Number: 5506587, Apr. 9, 1996.

[9] Tanenbaum, A.S., Computer Neworks. Prentice Hall, 4th edition, 2003.

[10] Rico Garcia, C., Lehner, A. \& Strang, T., A reliable MAC protocol for broadcast VANETs. V2VCOM 2008, ed. W. Altintas, Onur; Chen, Library of University of Twente, pp. 81-88, 2008.

[11] Rico Garcia, C., Lehner, A. \& Strang, T., Channel model for train to train communication using the $400 \mathrm{mhz}$ band. VTC2008-Spring, ed. V. Srinivasan, IEEE Conference eXpress Publishing, pp. 3082-3086, 2008. 